\title{
Visualization study of the influence of parapets on the flow around a train vehicle under cross winds
}

\author{
M. A. Barcala ${ }^{1} \&$ J. Meseguer ${ }^{2}$ \\ ${ }^{I}$ E.U.I.T. Aeronáutica, Universidad Politécnica de Madrid, Spain \\ ${ }^{2}$ IDR/UPM, E.T.S.I. Aeronáuticos, Universidad Politécnica de Madrid, \\ Spain
}

\begin{abstract}
Aerodynamic loads on train vehicles under cross winds are governed both by the shapes of the vehicles and of the surroundings. Aerodynamic loads due to cross winds are of paramount importance in the lateral equilibrium of the vehicle, in such a way that if the lateral wind speed becomes larger than a threshold value the overturning of the vehicle can take place. The degree of danger against overturning increases when the train is on a bridge, the reason being that the velocity in the atmospheric boundary layer grows as the height increases, leaving aside the fact that at ground level there may be other elements acting as windbreaks (mainly vegetation).

The effects of different types of parapets, either solid or porous, on the wind protection of a train vehicle on a bridge under cross wind have been visualized by smoke wind-tunnel tests. In addition the effects of several types of solid parapets on the side force and rolling moment acting on a two-dimensional model of the same high-speed train coach have been measured by wind-tunnel pressure measurements. Experimental results show that, as one could expect, solid parapets seem to be more effective than porous ones, and the aerodynamic loads decrease as the height of the windbreak increases.
\end{abstract}

Keywords: flow visualization, train aerodynamics, wind tunnel tests, overturning of train. 


\section{Introduction}

In the last years many efforts are being devoted to evaluate the cross-wind effects on the circulation of rail vehicles, in order to determine the limits of operation for high speed trains. Being a cross-disciplinary subject, the work must be subdivided in several tasks covering a wide range of aspects: aerodynamic tests in wind tunnels for the estimation of lateral loads on the vehicle and on the infrastructure, field measurements on the track, studies of aero-elastic phenomena induced on infrastructure components, effect of cross-winds on the vehicle dynamics (and specifically its influence on vehicle stability and the determination of overturning risk) and the evaluation of the level of risk (overturning, derailment and track lateral shift) by estimating the occurrence of combined effects of cross-wind and track singularities, amongst other.

The primary source of information for wind lateral loads on trains is windtunnel testing. Wind tunnel tests on train vehicles are generally performed through a static approach where the wind-train interaction is reproduced by means of a static model exposed to the wind-tunnel flow. Of course the use of a stationary train model subjected to wind perpendicular to the train side does not exactly reproduce the real situation. However, leaving aside some attempts made to consider the relative motion between train and ground (by moving the models through a wind tunnel [1,2] or by placing the train on a moving belt that simulates the ground displacement [3]), this technique seems to be suitable to analyze the relative effectiveness of parapets on the lateral aerodynamic loads on the train. Therefore, although this static method does not take into account the relative motion with respect to the ground, measurement on static models is the common, most widely used way to study train aerodynamics [4-6].

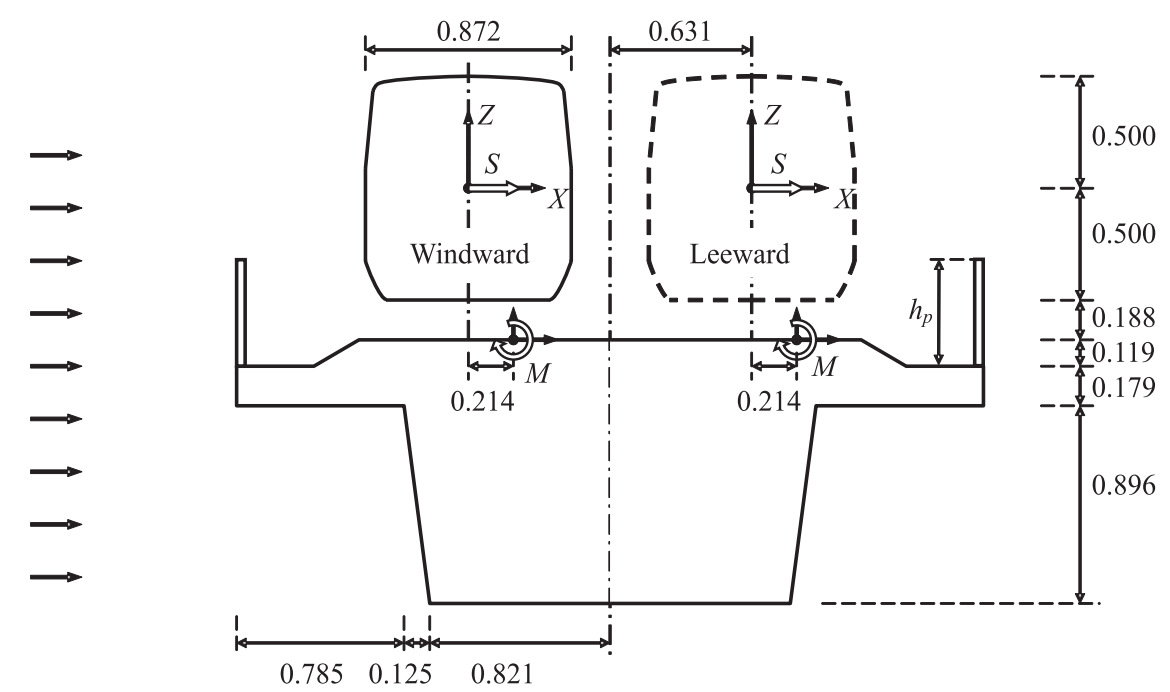

Figure 1: $\quad$ Sketch of the model cross section. All lengths have been made dimensionless with the coach height $H$. 
A way to decrease the lateral wind loads acting on a vehicle under lateral winds is to reduce the wind effects by using fences (either solid or porous). The sheltering ability of different types of parapets to prevent lateral wind loads on a train on a bridge was evaluated both by flow visualization and by pressure measurement on a static two-dimensional train model. The tested configuration was a typical high-speed train vehicle (a Talgo coach) on a double-track bridge deck equipped with different types of vertical parapets, either solid or porous (figure 1). The results corresponding to non-vertical solid parapets equipped with eaves have been published elsewhere [6].

Besides wind loads measurements, smoke visualization of the flow around the train model on the bridge has been performed to clarify the flow morphology around the coach. It must be pointed out that although visualization tests are only qualitative (because of the low value of Reynolds number), in the case of wedged bodies they provide a good estimation of the real flow, because in wedges bodies boundary layer separation takes place at forward body edges at low Reynolds numbers, so that the flow pattern becomes almost Reynolds number independent.

\section{Experimental set-up}

Visualization of the flow around the model has been performed by using a small two-dimensional smoke wind tunnel. The working section is $0.4 \mathrm{~m}$ high, $0.04 \mathrm{~m}$ wide and $0.6 \mathrm{~m}$ long.

The model scale was $1 / 60$. The model represents the cross-sectional shape of the high-speed Talgo passenger coach. Details on the train surface, such as the windows, were not reproduced. The bridge is a typical double track cross-section bridge (figure 1). Both vehicle model and bridge model are $0.04 \mathrm{~m}$ wide, so that they span the test chamber width. Since the bridge cross-section corresponds to a double-track bridge deck, two positions of the vehicle, either at the windward or the leeward track, were considered.

Parapets are vertical walls of different dimensionless height, $h_{p}=H_{p} / H, H_{p}$ being the parapet height and $H$ the vehicle height. Five dimensionless heights have been considered $\left(h_{p}=0.30,0.45,0.60,0.75\right.$ and 0.90$)$, and for each one of these heights five different porosities were tested $(\phi=85 \%, 70 \%, 50 \% 25 \%$ and $0 \%$ ). Parapets with large porosity, $\phi=85 \%$ are made with horizontal $1 \mathrm{~mm}$ diameter wires adequately spaced, $\phi=70 \%$ parapets are perforated plates and $\phi=50 \%$ and $\phi=25 \%$ were made of commercial meshes of the appropriate porosity; finally, $\phi=0 \%$ parapets are solid walls.

Additional tests were carried out to get a quantitative measure of the effectiveness of vertical parapets to reduce lateral aerodynamic loads on trains. For these new tests a second wind tunnel with $1.80 \mathrm{~m} \times 1.50 \mathrm{~m}$ cross-section and new models with scale $1 / 27$ were used (their shapes being equal to the ones used in visualization tests). Reynolds number was close to $7 \times 10^{5}$. No atmospheric boundary layer has been reproduced in the experiments because for the configuration under consideration (a train on a bridge) vertical velocity gradients become negligible once the characteristic height of the obstacle and the 
characteristic height of the atmospheric boundary layer are taken into account. The wind velocity profile at the model test section was uniform within $\pm 1 \%$, the turbulence intensity being around $4 \%$.

In this case both vehicle model and bridge model are $1.5 \mathrm{~m}$ long, so that they span the test chamber width. The vehicle model is equipped with 30 pressure taps distributed in the middle model cross-section. Each pressure tap consists of a brass tube, of $1 \mathrm{~mm}$ inner diameter, which is connected by $1 \mathrm{~mm}$ inner diameter plastic tube to a pressure scanner from Scanivalve Corp., pressure outputs were sampled at $20 \mathrm{~Hz}$ during 12.5 seconds for each measurement. The pressure coefficient is defined as usual, $c_{p \mathrm{n}}=\left(p_{\mathrm{n}}-p\right) / q$, where $p_{\mathrm{n}}$ is the mean pressure measured on tap $\mathrm{n}$, and $p$ and $q=\rho U^{2} / 2$ are the static and dynamic pressures upstream of the model, respectively, $\rho$ is the air density and $U$ the unperturbed flow velocity. The Reynolds number is, $\mathrm{Re}=U H / v, v$ being the kinematic viscosity of air $\left(v=1.5 \times 10^{-5} \mathrm{~m}^{2} / \mathrm{s}\right)$.

\section{Experimental results}

Some of the results of the visualization test are shown in figures 2, 3, 4 and 5. In figure 2 the flow pattern around the model with the coach either in the windward or the leeward tracks is shown (this is the case of reference, without parapets). Observe that there are not large differences between both pictures, streamlines being almost equal in both cases. There is, however, a subtle difference in the streamline just impinging in the front of the bridge deck. With the coach in the windward position such a streamline is entirely deflected downwards, whereas in the leeward case the streamline bifurcates, and part of the smoke is deflected upwards. However, such a difference mainly affects to the lift force on the whole bridge-vehicle configuration.

In the case of solid parapets $(\phi=0 \%)$ the flow configuration becomes notably changed as the height of the wall increases (figure 5), the differences being remarkable when the vehicle is on the windward track. Observe that the model is practically shielded when $h_{p} \approx 0.6$. Such situation is even stressed when the coach is on the leeward track: the parapet gives rise to a wide wake where the model is immersed, thus decreasing the aerodynamic loads.
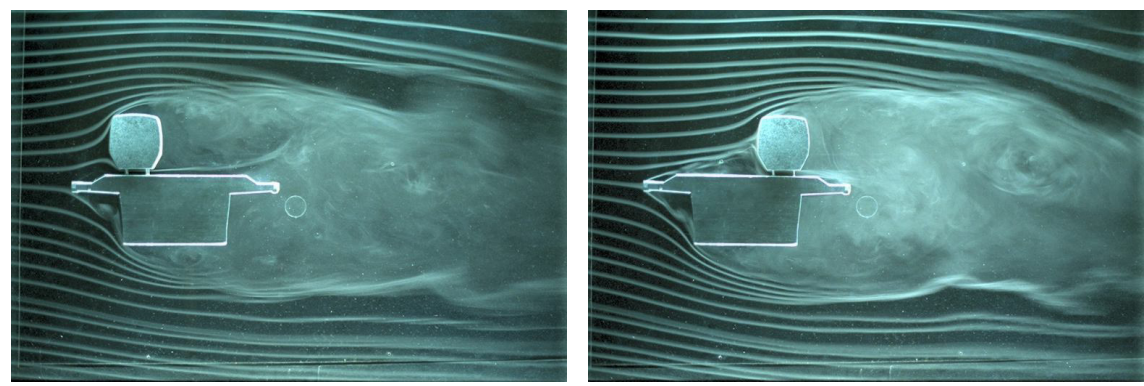

Figure 2: $\quad$ Flow streamlines around both the train coach and bridge models in the case of a bridge without parapets. 

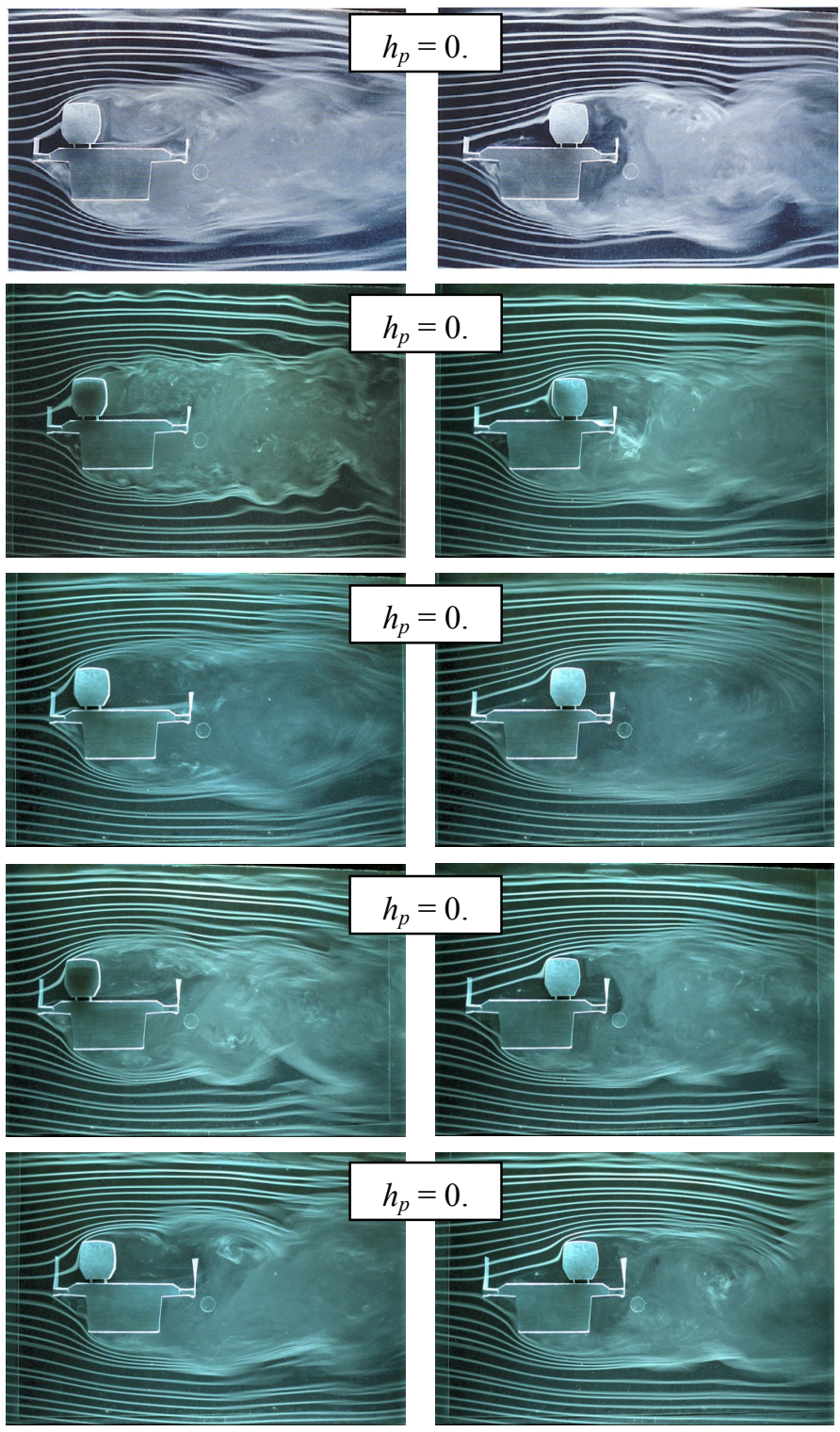

Figure 3: $\quad$ Flow streamlines around both the train coach and bridge models in the case of bridge with porous parapets $(\phi=90 \%)$. The dimensionless parapet heights $h_{p}$ are indicated in the inserts Very porous parapets (figure 3 ) do not alter the flow pattern with respect to the flow pattern obtained without protecting walls. Of course the shielding effect increases as the height of the parapets grows, but the differences are not too large. Such behaviour is almost the same for medium porosity parapets (figure 4). 

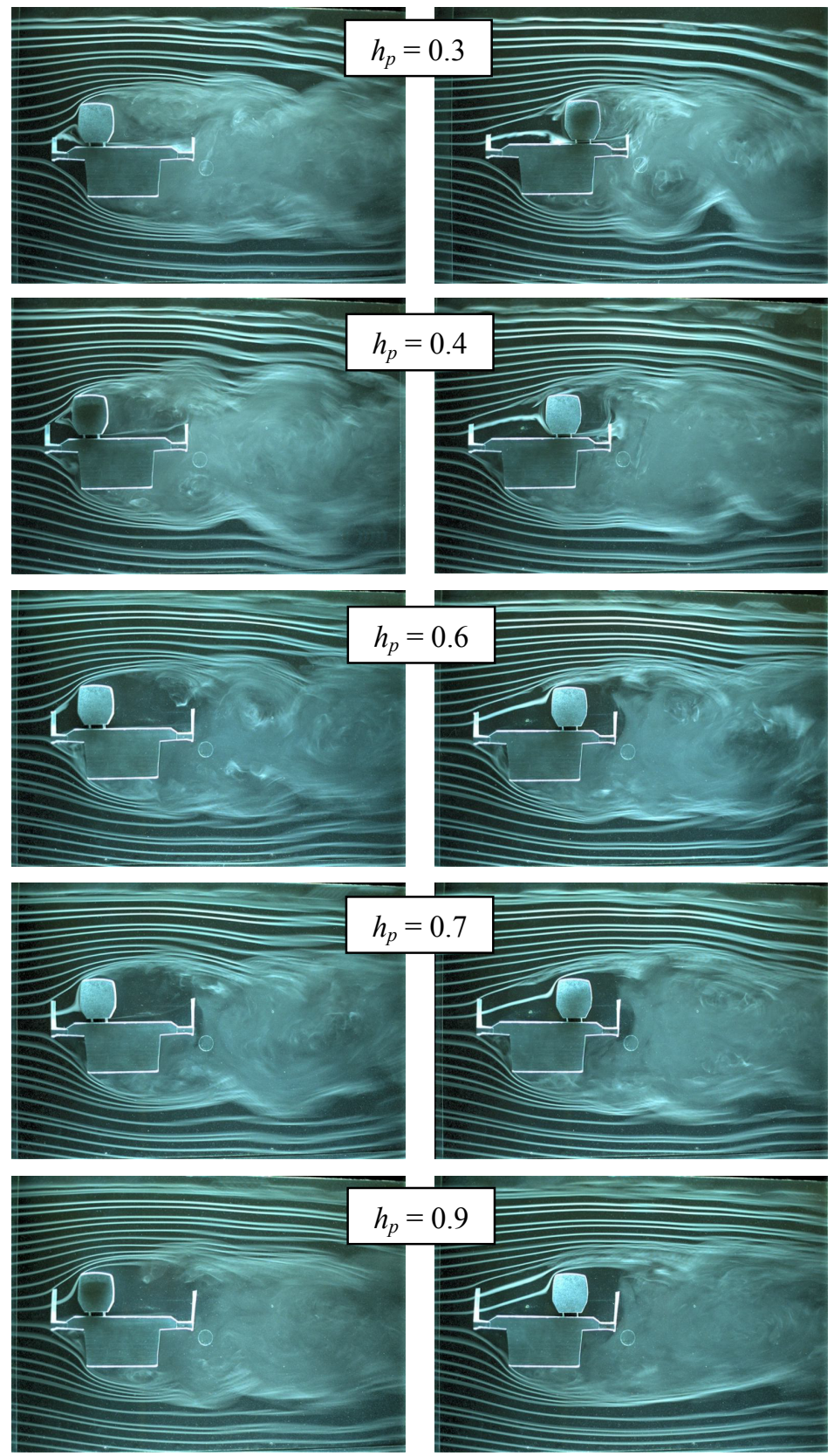

Figure 4: $\quad$ Flow streamlines around both the train coach and bridge models in the case of bridge with porous parapets $(\phi=50 \%)$. The dimensionless parapet heights $h_{p}$ are indicated in the inserts. 

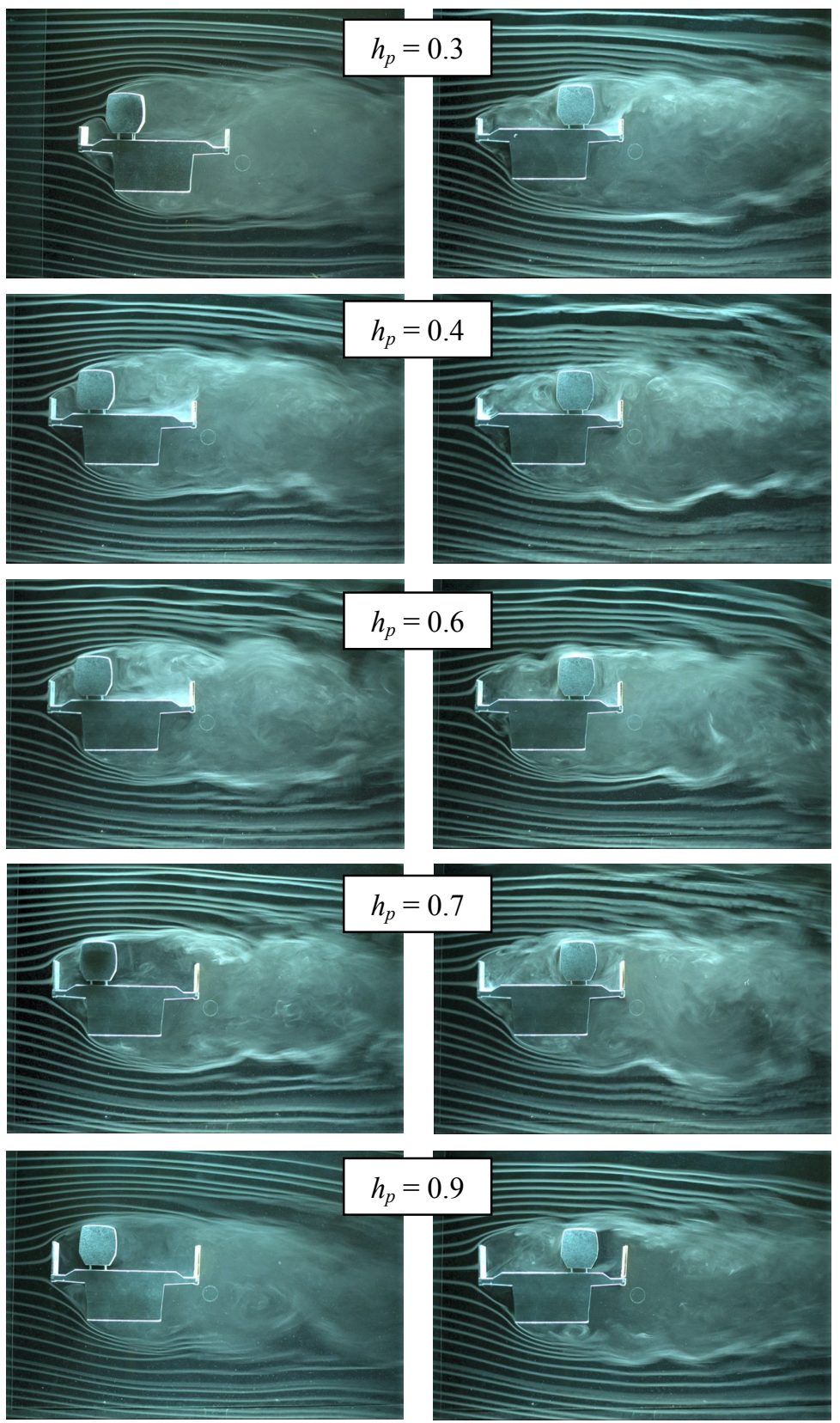

Figure 5: Flow streamlines around both the train coach and bridge models in the case of bridge with solid parapets $(\phi=0 \%)$. The dimensionless parapet heights $h_{p}$ are indicated in the inserts. 
From the measured pressure distributions the side force and the overturning moment coefficients were calculated (figure 1). These coefficients are defined as usual: $c_{S}=S /(q H), c_{M}=M /(q H C), S$ being the aerodynamic side force, $M$ the overturning moment (both force and moment are per unit length) and $C$ the vehicle width $(C=0.872 H)$.

Some experimental results are shown in figure 6 , where the variation with the dimensionless parapet height, $h_{p}$, of the normalised side force coefficients and the normalised moment coefficients are represented. These results correspond to the coach in the windward track. The normalised load coefficients, $c_{S} / c_{S w}$ or $c_{M} / c_{M w}$, are defined as the ratio of the load coefficients $\left(c_{S}\right.$ or $\left.c_{M}\right)$ to the same load coefficients measured without any parapet $\left(c_{S w}\right.$ or $\left.c_{M w}\right)$.

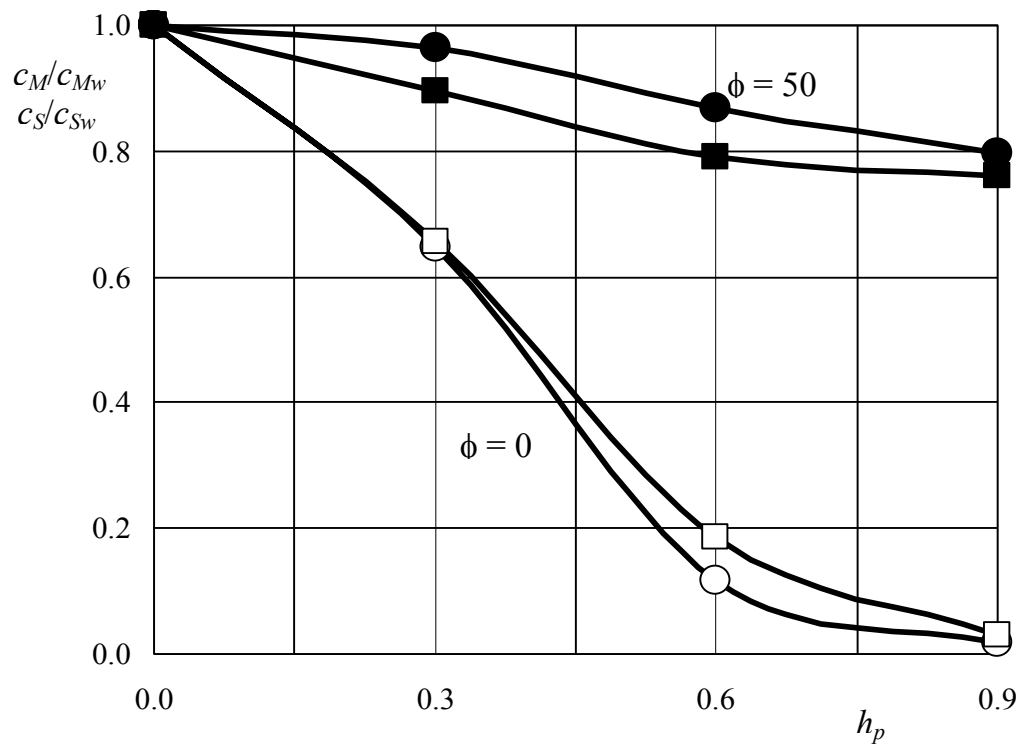

Figure 6: Variation with the dimensionless height of the parapet, $h_{p}$, of the ratio $c_{S} / c_{S w}$ between the aerodynamic side force coefficient, $c_{S}$, and the same side force coefficient measured without any parapet, $c_{S w}$ (circles), and the ratio $c_{M} / c_{M w}$ between the overturning moment coefficient, $c_{M}$, and the same moment coefficient measured without any parapet, $c_{M w}$ (squares). The results correspond to the train on windward track on the bridge. The symbols identify the parapet: solid parapets (open symbols), and porous parapets with porosity $\phi=50 \%$ (closed symbols)

According to the measured results, a given parapet seems to increase the effectiveness to reduce the wind loads on the vehicle as its relative height increases (irrespective of whether the train is on the windward or on the leeward track [6]). Obviously such behaviour is explained because of the separation of the boundary layer starting at the stagnation point existing at the windward face 
of the obstacle, which separates both at the bottom of the bridge structure and at the upper edge of the parapet, thus forming a separation bubble downstream. Since the size of the separation bubble increases as the parapet height grows, the vehicles placed downstream of the parapet became more and more protected against incident wind as they are placed more and more inside the separated bubble (this also explains why loads are even smaller when the vehicle is in the leeward track).

Concerning the overturning moments, since the cross-section shape of a train coach is not designed to produce lift (although some lift force is produced because the flow accelerates at the train roof and decelerates at the bottom, these lift forces are small when compared with side forces), the overturning moment coefficients behave almost exactly the same as the side force coefficients.

\section{Conclusions}

The effects of different types of parapets, either solid or porous, on the wind protection of a train vehicle on a bridge under cross wind have been visualized by smoke visualization. The effect of porosity is almost negligible when the value of this parameter is small enough, so that vertical parapets become effective to decrease wind loads only when they are solid or almost solid $(\phi \cong 0 \%)$.

The shielding effectiveness of vertical parapets has been also experimentally analyzed by measuring the pressure distributions on a train coach model placed downstream of the parapets. Experimental results show that increasing the height of the parapets improves the shielding effectiveness of the wind barriers. In the case of solid parapets a very drastic reduction of the wind load coefficients results provided the parapet height is large enough.

\section{References}

[1] C.J. Baker, Train aerodynamic forces and moments from moving model experiments, Journal of Wind Engineering and Industrial Aerodynamics, 24, 227-251, 1986.

[2] C.J. Baker, N.D. Humphreys, Assessment on the adequacy of various wind tunnel techniques to obtain aerodynamic data for ground vehicles in cross winds, Journal of Wind Engineering and Industrial Aerodynamics, 60, 4968, 1996.

[3] H. Kwon, Y. Park, D. Lee, M. Kim, Wind tunnel experiments on Korean high-speed trains using various ground simulation techniques, Journal of Wind Engineering and Industrial Aerodynamics, 89, 1179-1195, 2001.

[4] S. Sanquer, C. Barré, M. Dufresne de Virel, L.M. Cléon, Effect of cross winds on high-speed trains: development of a new experimental methodology, Journal of Wind Engineering and Industrial Aerodynamics, 92, 535-545, 2004. 
[5] M. Suzuki, K. Tanemoto, T. Maeda, Aerodynamic characteristics of train/vehicles under cross winds, Journal of Wind Engineering and Industrial Aerodynamics, 91, 209-218, 2003.

[6] M.A. Barcala, J. Meseguer, An experimental study of the influence of parapets on the aerodynamic loads under cross wind on a two-dimensional model of train vehicle on a bridge, Proc. Instn. Mech. Engrs, Part F, Journal of Rail and Rapid Transit, 221, 487-494, 2007. 